\title{
CLINICAL PERFORMANCE OF A NOVEL DAILY DISPOSABLE SOFT CONTACT LENS
}

Stacie Cummings, OD, ${ }^{1 *}$ Bradley Giedd, OD, MS, FAAO, ${ }^{2}$ Christopher Pearson, $\mathrm{OD}^{3}$

${ }^{1}$ Alcon, Johns Creek, GA USA, ${ }^{2}$ Maitland Vision Center, Maitland, FL USA

${ }^{3}$ OMEGA Vision Center, Longwood, FL USA.

*Corresponding author: Stacie Cummings, OD: stacie.cummings@alcon.com.

Submitted: May 5, 2020. Accepted: October 9, 2020. Published: December 11, 2020.

\section{ABSTRACT}

\section{Background and Objective}

Verofilcon A (PRECISION1®; Alcon) silicone hydrogel daily disposable contact lenses were designed to address clinical issues associated with new wearer discontinuation, including vision quality, sustained comfort, and ease of handling. The present study assessed the objective and subjective performance of verofilcon A contact lenses in subjects needing optical correction for refractive ametropia.

\section{Material and Methods}

Subjects aged 18 years and older with non-diseased eyes, a history of successful soft contact lens wear for distance correction in both eyes during the previous 3 months, and a best spectacle-corrected visual acuity $20 / 25$ or better with a manifest cylinder of $\leq 0.75$ diopters in each eye were recruited. The primary endpoint was distance visual acuity. Secondary endpoints included subjective ratings of the overall vision, comfort, handling, and safety endpoints, including adverse events and biomicroscopy/slit-lamp examination findings.

\section{Results}

This study recruited 70 subjects (140 eyes), aged 20-48 years, who were predominantly white (93\%), nonHispanic/Latino (94\%), and female (73\%); one subject was discontinued due to a change in residence. Of the 140 eyes, $132(94.3 \%)$ had 20/20 or better vision with their habitual contact lenses. Similarly, of the 138 eyes that completed the study, $136(98.6 \%)$ had 20/20 or better vision at dispensation of verofilcon A lenses, and $132(95.7 \%)$ had 20/20 or better vision at 3 months. Mean \pm SD subjective ratings of overall vision, overall comfort, and overall handling, each on a 10-point scale, were $9.4 \pm 0.8,9.4 \pm 0.8$, and $9.2 \pm$ 1.3, respectively, at lens dispensing, and $9.4 \pm 0.9,9.5 \pm 0.8$, and $9.2 \pm 1.0$, respectively, at 3 months. Mean \pm SD daily wear times at 1 week and 3 months were $14.71 \pm 1.85$ and $15.05 \pm 1.27$ hours, respectively,

J Cont Lens Res Sci Vol 4(2):e23-e30; December 11, 2020

This article is distributed under the terms of the Creative Commons Attribution-Non

Commercial 4.0 International License. (c) Cummings et al 
with $68.1 \%$ at 1 week and $73.9 \%$ at 3 months wearing verofilcon A lenses for $14-18$ hours per day. None of the subjects experienced a severe adverse event or discontinued lens wear, with biomicroscopy/slit-lamp examination showing no evidence of corneal edema or chemosis.

\section{Conclusion}

Subjects wearing verofilcon A lenses had visual acuity similar to that when wearing their habitual lens. Verofilcon A lenses were highly rated for vision quality, comfort, and handling. Verofilcon A lenses may be an option for subjects with refractive ametropia who desire lenses that can be worn for $>14$ hours per day and are easy to handle.

Key Words: verofilcon A, visual acuity, comfort, wear times

\section{INTRODUCTION}

Delefilcon A (DAILIES TOTAL1 ${ }^{\circledR}$; Alcon) contact lenses were the first water gradient contact lens introduced in the US, with $33 \%$ water content at the core and nearly $100 \%$ water content at the surface. Verofilcon A (PRECISION $1^{\circledR}$; Alcon) contact lenses are silicone hydrogel daily disposable contact lenses designed to address the key clinical issues associated with new wearer discontinuation: vision quality, sustained comfort, and ease of handling. ${ }^{1}$ SMARTSURFACE $\AA$ technology, developed for the production of verofilcon $\mathrm{A}$ lenses, provides a permanent, micro-thin layer of moisture that surrounds the verofilcon A core material. This results in a lens comprised of a $51 \%$ water silicone hydrogel core encased in a thin hydrogel surface with more than $80 \%$ water.

The verofilcon $\mathrm{A}$ lens manufacturing process uses a proprietary sequence of $\mathrm{pH}$ and temperature-controlled baths to embed the surface to the lens permanently. The first bath slightly expands the lens, creating small, shallow pores in the lens surface. Hydrophilic polymers in the bath penetrate the open pores and lock into place. The result is a micro-thin layer of moisture ( $\sim 2$ microns), which exceeds $80 \%$ water content at the lens surface. Verofilcon A lenses have a modulus of $0.6 \mathrm{MPa}$ and oxygen transmissibility of $100 \mathrm{Dk} / \mathrm{t}$ at -3.00 D. These lenses also provide Class I ultraviolet filtration, blocking $>90 \%$ of ultraviolet A and $>99 \%$ of ultraviolet $\mathrm{B}$ rays. The base curve and diameter of these lenses are $8.3 \mathrm{~mm}$ and $14.2 \mathrm{~mm}$, respectively.

The present study assessed the objective and subjective performance of verofilcon A contact lenses in subjects needing optical correction for refractive ametropia.

\section{METHODS}

\section{Study Population}

This manuscript reports the results of one arm of a prospective, randomized, stratified (by corneal curvature radius), controlled, double-masked, parallel-group clinical trial comparing daily disposable verofilcon A contact lenses with daily disposable delefilcon A contact lenses in subjects aged $\geq 18$ years with nondiseased eyes and in need of optical correction for refractive ametropia. Other eligibility requirements included a history of successful soft contact lens wear for distance correction in both eyes during the previous 3 months for a minimum of 5 days per week and 8 hours per day, and a best spectacle-corrected visual acuity of $20 / 25$ or better with a manifest cylinder of $\leq 0.75$ diopters (D) in each eye.

Subjects were excluded if they had any anterior segment infection, inflammation, abnormality, or ocular or systemic disease that contraindicated contact lens wear or if they were using systemic or ocular medications for which contact lens wear was contraindicated. Subjects were also excluded if they had any history of ocular or intraocular surgery, biomicroscopy find-ings indicative of corneal vascularization; or current or previous history of herpetic keratitis. Subjects who were current or previous wearers of delefilcon A lenses and those who routinely slept in their lenses for at least one night per week for the 3 months before enrollment were also excluded.

Subjects at six sites in the United States were randomized 2:1 to verofilcon A or delefilcon A lenses. Seven scheduled visits were planned: a screening/ baseline visit, during which the subjects were randomized, lenses were fitted and lens power selected, a

J Cont Lens Res Sci Vol 4(2):e23-e30; December 11, 2020

This article is distributed under the terms of the Creative Commons Attribution-Non

Commercial 4.0 International License. (C) Cummings et al 
dispensing visit; and follow-up visits at 1 and 2 weeks and at 1,2, and 3 months. This report describes the 3 -month outcomes in subjects randomized to verofilcon A lenses.

An institutional review board approved this trial, which complied with the Declaration of Helsinki. All subjects provided written informed consent. This trial was registered on ClinicalTrials. gov as number NCT03305770.

\section{Endpoints}

The primary endpoint was distance visual acuity. Visual acuity was measured in each eye using Snellen charts under dimmed room illumination, with no extraneous light sources shining on the chart. All measurements were converted to the logMAR scale. Snellen was used for categorical reporting and logMAR for numerical reporting.

Exploratory endpoints included subjective ratings of the overall vision, comfort, and handling, which subjects rated on a scale of 1 (Poor) to 10 (Excellent). Subjects rated overall vision at each study visit based on their quality of vision over a full day. Subjects rated overall comfort at each study visit as the average comfort of lenses over a full day since their previous study visit. Subjects rated overall handling at each study visit based on the ease of inserting and removing the lenses, including determining whether the lens is inside-out and positioning it for insertion. Safety outcomes recorded at each visit included adverse events (AEs) and biomicroscopy/slit-lamp examination findings.

\section{Statistical Analysis}

The analysis sets consisted of all subjects/eyes in the enrolled dispensed set that completed the study. Descriptive statistics were provided for demographics and subject characteristics and the effectiveness and safety endpoints. Descriptive statistics for the visual acuity assessments included $\mathrm{N}$ and \% for Snellen categories; and $\mathrm{N}$, mean, $\mathrm{SD}$, median, min, and max for the converted logarithmic minimum angle of resolution ( $\log$ MAR) values. Descriptive statistics for the subjective ratings included $\mathrm{N}$ and \% for categorical variables; and $\mathrm{N}$, mean, $\mathrm{SD}$, median, minimum, and maximum for continuous variables. All statistical analyses were performed using SAS ${ }^{\circledR}$ software (SAS Institute Inc., Cary, NC).

\section{RESULTS}

\section{Characteristics of the Study Subjects}

A total of 70 subjects were dispensed verofilcon A lenses. Of these, one subject ( 2 eyes) was discontinued because of a change in residence. Thus, complete data

TABLE 1 Verofilcon A Patient Demographics (All Enrolled Subjects)

\begin{tabular}{|l|c|}
\hline Age, $y$ & $\begin{array}{c}\text { Subjects } \\
(\mathbf{n}=\mathbf{7 0})\end{array}$ \\
$\begin{array}{l}\text { Mean (SD) } \\
\text { Range }\end{array}$ & $34.3(6.3)$ \\
Sex, n (\%) & 20,48 \\
Male & $19(27.1)$ \\
Female & $51(72.9)$ \\
\hline Race, n (\%) & $65(92.9)$ \\
White & $3(4.3)$ \\
Black/African American & $0(0)$ \\
American Indian/Alaska & $1(1.4)$ \\
Native & $1(1.4)$ \\
Asian & $4(5.7)$ \\
Other & $66(94.3)$ \\
\hline Ethnicity, n (\%) & \\
Hispanic/Latino & Not Hispanic/Latino \\
\hline
\end{tabular}

$S D=$ standard deviation .

J Cont Lens Res Sci Vol 4(2):e23-e30; December 11, 2020

This article is distributed under the terms of the Creative Commons Attribution-Non

Commercial 4.0 International License. (C) Cummings et al 
for wear of verofilcon A lenses were obtained for 138 eyes. The subject population was predominantly white (93\%), non-Hispanic/Latino (94\%), and female (73\%). Subjects ranged in age from 20 to 48 years with a mean of 34.3 years. Demographic data of the subjects dispensed verofilcon A lenses are shown in Table 1.

Nearly all subjects had 20/20 vision or better with their habitual contact lenses. The mean habitual contact lens power (sphere) for subjects was $-3.38 \mathrm{D}$ (standard deviation, 1.37). Habitual lens prescription data are given in Table 2 . The mean study lens power (sphere) at the dispensing visit and study completion were similar ( $-3.38 \mathrm{D}$ [1.36], ranging from -1.00 to $-5.75 \mathrm{D}$ at the dispensing visit and $-3.40 \mathrm{D}$ [1.36], ranging from -1.00 to $-5.75 \mathrm{D}$ at study completion).

\section{Efficacy of Verofilcon A Lenses}

The verofilcon A lenses conferred visual acuity of $20 / 15$ or $20 / 20$ in $98.5 \%$ of subjects at the dispense visit and in $95.7 \%$ of subjects at the 3-month follow-up visit (Table 3). All subjects had 20/30 or better visual acuity at all study visits.

Subjects gave high ratings for overall vision on a 10-point scale (1 [poor] to 10 [excellent]) (Figure 1A). The mean score at dispense was 9.4 (0.8). Subjects continued to rate overall vision high throughout the study. At the 3-month follow-up visit, the mean score was $9.4(0.9)$.

Overall lens comfort on a 10-point scale (1 [poor] to 10 [excellent]) was also highly rated by subjects who wore verofilcon A lenses (Figure 1B). At the dispensing visit, the mean score was 9.4 (0.8). Subjective ratings for overall comfort were maintained throughout the study, and the mean score at the 3-month follow-up visit was $9.5(0.8)$.

High subjective ratings on a 10-point scale (1 [poor] to 10 [excellent]) were also given for overall

TABLE 2 Baseline Visual Acuity and Subjective Refraction with Habitual Contact Lenses (All Enrolled Eyes)

\begin{tabular}{|c|c|}
\hline & $\begin{array}{c}\text { Number of Eyes } \\
(n=140)\end{array}$ \\
\hline \multicolumn{2}{|l|}{ Visual acuity } \\
\hline Snellen category, n (\%) & 140 \\
\hline $20 / 15$ & $40(28.6)$ \\
\hline $20 / 20$ & $92(65.7)$ \\
\hline $20 / 25$ & $8(5.7)$ \\
\hline $\begin{array}{l}\log M A R \\
\text { Mean (SD) } \\
\text { Range }\end{array}$ & $\begin{array}{l}-0.03(0.06) \\
(-0.12,0.10)\end{array}$ \\
\hline \multicolumn{2}{|l|}{ Sphere, D } \\
\hline $\mathrm{n}$ & 140 \\
\hline Mean (SD) & $-3.38(1.37)$ \\
\hline Range & $(-6.00,-0.75)$ \\
\hline \multicolumn{2}{|l|}{ Cylinder, D } \\
\hline $\mathrm{n}$ & 140 \\
\hline Mean (SD) & $-0.00(0.02)$ \\
\hline Range & $(-0.25,0.00)$ \\
\hline \multicolumn{2}{|l|}{ Axis } \\
\hline $\mathrm{n}$ & 1 \\
\hline Mean (SD) & $140.0(0.0)$ \\
\hline Range & $(140,140)$ \\
\hline \multicolumn{2}{|l|}{ ADD Power } \\
\hline $\mathrm{n}$ & 140 \\
\hline Mean (SD) & $0.00(0.00)$ \\
\hline Range & $(0.00,0.00)$ \\
\hline \multicolumn{2}{|c|}{$D=$ diopter $; \log M A R=$ logarithmic minimum angle of resolution; $S D=$ standard deviation } \\
\hline \multicolumn{2}{|c|}{$\begin{array}{l}\text { J Cont Lens Res Sci Vol 4(2):e23-e30; December 11, } 2020 \\
\text { This article is distributed under the terms of the Creative Commons Attribution-Non } \\
\text { Commercial } 4.0 \text { International License. @ Cummings et al }\end{array}$} \\
\hline
\end{tabular}


TABLE 3 Visual Acuity with Verofilcon A Lenses

\begin{tabular}{|c|c|}
\hline \multicolumn{1}{|c|}{ Visit } & $\begin{array}{c}\text { Number of Eyes } \\
(\mathbf{n}=\mathbf{1 3 8})\end{array}$ \\
\hline $\begin{array}{c}\text { Dispense } \\
\text { Snellen category, n (\%) }\end{array}$ \\
$20 / 15$ & $74(53.6)$ \\
$20 / 20$ & $62(44.9)$ \\
$20 / 25$ & $2(1.4)$ \\
logMAR & \\
Mean (SD) & $-0.07(0.07)$ \\
Range & $(-0.12,0.10)$ \\
\hline 3-Month follow-up & \\
Snellen category, n (\%) & $63(45.7)$ \\
$20 / 15$ & $69(50.0)$ \\
$20 / 20$ & $6(4.3)$ \\
$20 / 25$ & \\
logMAR & $-0.05(0.07)$ \\
Mean (SD) & $(-0.12,0.10)$ \\
\hline
\end{tabular}

$\log M A R=$ logarithmic minimum angle of resolution; $S D=$ standard deviation.

handling of the verofilcon A lenses (Figure 1C). At the dispensing visit, the mean score for overall handling was 9.2 (1.3). Subjective ratings for overall handling remained high throughout the study. At the 3-month follow-up, the mean score was 9.2 (1.0).

Data for daily wear times are shown in Figure 2. At the 1-week follow-up visit, the mean daily wear time was 14.71 hours (1.85), and at the 3-month follow-up visit, the mean daily wear time was 15.05 hours (1.27). Over two-thirds $(68.1 \%)$ of subjects at 1 week and nearly three-quarters $(73.9 \%)$ at 3 months wore these lenses for 14-18 hours per day.

\section{Safety and Tolerability}

Two subjects were discontinued during this study $(2.8 \%)$, and only one of these subjects discontinued wearing the verofilcon A lenses during the study (1.4\%). One subject discontinued before being dispensed the study lenses due to an investigator's decision, and the second discontinued after study lenses were dispensed due to relocation. None of the subjects experienced a severe adverse event or discontinuation. There were no reports of corneal edema or chemosis on biomicroscopy/slit-lamp examination. This study's findings demonstrated that these soft contact lenses were safe, based on the low rates of AEs and the absence of any other clinically significant safety findings.

\section{DISCUSSION}

In a recently published study of new contact lens wearers in which $22 \%$ of subjects permanently discontinued wearing contact lenses during the first year, half of these discontinued during the first three months. ${ }^{2}$ The primary reason for discontinuation given by the new wearers was low vision quality, followed by poor comfort and difficulty in handling.

Verofilcon A lenses were designed to address these clinical performance issues. Clinical evaluations showed that verofilcon A lenses provided a high level of visual acuity for correction of refractive ametropia in adults, with more than $95 \%$ of eyes corrected to 20/20 or better. Mean subjective ratings for overall vision, comfort, and handling were greater than 9 out of a possible 10. All study subjects had 20/30 or better visual acuity at all visits, with most having visual acuity of $20 / 20$ or $20 / 15$ at lens dispensation and 3-month follow-up.

Because approximately one-third of all contact lens fits are for new contact lens wearers, ${ }^{4}$ providing new contact lens wearers with a positive experience benefits both subjects and eye care practitioners. Patients gain the advantages of greater vision quality and more freedom to engage in sports and other activities. They may also develop more confidence

J Cont Lens Res Sci Vol 4(2):e23-e30; December 11, 2020

This article is distributed under the terms of the Creative Commons Attribution-Non

Commercial 4.0 International License. (c) Cummings et al 
FIG. 1 Overall subjective ratings of (A) vision, (B) comfort, and (C) handling at the dispense visit and the 3 -month follow-up visit (on scales of $1-10$, where 1 = poor and $10=$ excellent).
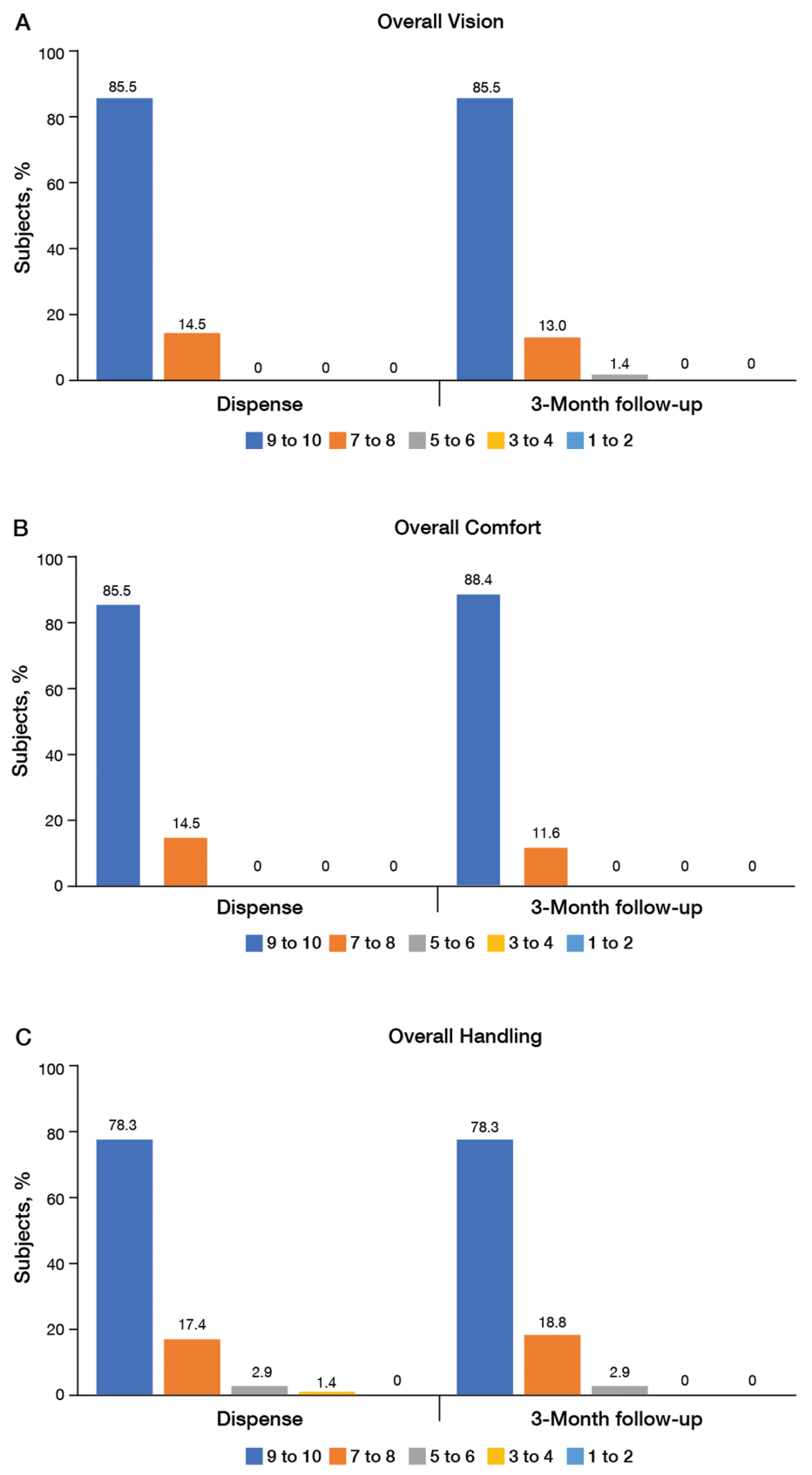

in and loyalty to their eye care provider, leading to referrals to friends and family members. Eye care practices benefit from the increased patient volume, greater frequency of examinations, and higher contact lens and professional fee revenue.
The duration of wear for the verofilcon A lenses also supports these new lenses' high degree of comfort. Contact lens discomfort frequently results in reduced wear time. ${ }^{2}$ The high oxygen transmissibility of silicone hydrogel contact lenses has been associated with 
FIG. 2 Subject-reported daily lens wear times (hours) at the 1-week and 3-month follow-up visits.

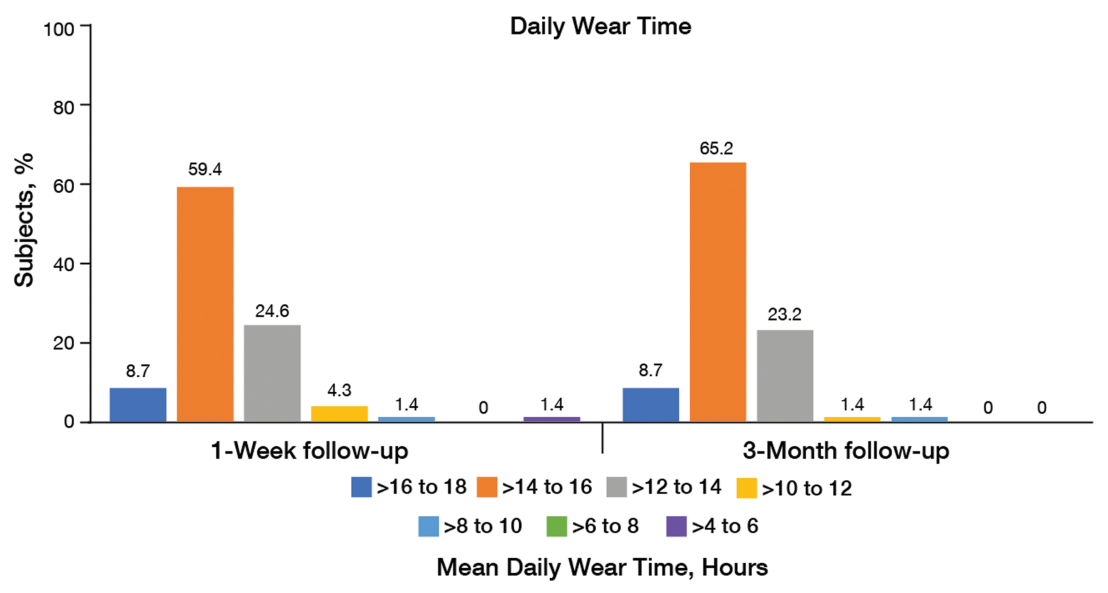

improved patient symptoms of comfort, including dryness and wear time..$^{5-8}$ The overall high ratings of verofilcon A lenses' comfort are therefore consistent with their high oxygen transmissibility $(100 \mathrm{Dk} / \mathrm{t}$ at $-3.00 \mathrm{D})$. These lenses also provide Class I ultraviolet filtration, blocking $>90 \%$ of ultraviolet A and $>99 \%$ of ultraviolet $\mathrm{B}$ rays.

A similar study assessed the reactions of 129 new contact lens wearers and 172 experienced wearers of daily disposable contact lenses to wearing verofilcon A lenses for 1 week. ${ }^{9}$ Preferences of new contact lens wearers reflected improvements in their day-to-day quality of life. Of the 129 new contact lens wearers, $91 \%$ agreed that verofilcon A lenses provided them the opportunity to be spectacle-free, $79 \%$ agreed that these lenses were great for starting their contact lens wearing experience, and $70 \%$ agreed that they would continue to wear these lenses in future. Also, $78 \%$ agreed that the clear vision provided at the end of the day was similar to that provided at the beginning of the day, $79 \%$ agreed that these lenses provided clear vision while looking at digital devices, $75 \%$ agreed that they were comfortable to wear during the day, $82 \%$ agreed that they were easy to insert, and $72 \%$ agreed that they were easy to remove. Of the 172 experienced contact lens wearers, $81 \%$ preferred verofilcon A lenses to their habitual lenses, due primarily to long-lasting comfort and vision. ${ }^{9}$

\section{CONCLUSION}

This study showed that verofilcon A lenses conferred visual acuity nearly equivalent to the subjects' entering visual acuity. Furthermore, subjects consistently provided high ratings for vision quality, comfort, and handling. Verofilcon A lenses are an option for new and experienced contact lens wearers with refractive ametropia who desire lenses that can be worn for $>14$ hours per day and are easy to handle.

\section{SUBMISSION DECLARATION}

Portions of the study results have been presented previously at the American Academy of Optometry annual meeting, held on October 23-26, 2019, in Orlando, Florida, USA.

\section{FUNDING}

This study was funded by Alcon Research, LLC. $\mathrm{SC}$ is an employee of Alcon Research, LLC. BelMed Professional Resources, Inc, provided editorial support, which was contracted and funded by Alcon.

\section{ACKNOWLEDGMENTS}

The authors would like to thank Bradley N. Hines, OD, Wilson W. Movic, OD, FAAO, Peter Van Horn, OD, and Eric White, OD for their contributions as investigators for this study.

J Cont Lens Res Sci Vol 4(2):e23-e30; December 11, 2020

This article is distributed under the terms of the Creative Commons Attribution-Non

Commercial 4.0 International License. (c) Cummings et al 


\section{REFERENCES}

1. Nichols JJ, Willcox MD, Bron AJ, et al. The TFOS International Workshop on Contact Lens Discomfort: executive summary. Invest Ophthalmol Vis Sci 2013;54(11):TFOS7-13.

2. Sulley A, Young G, Hunt C, et al. Retention rates in new contact lens wearers. Eye Contact Lens 2018;44 Suppl 1:S273-82.

3. Downie LE, Craig JP. Tear film evaluation and management in soft contact lens wear: a systematic approach. Clin Exp Optom 2017;100(5):438-58.

4. Growth From Knowledge. Daily Contact Lenses Surpass Monthlies In US Sales, Account For 38\% Of Market. 2017 [Internet]. Available at: https://www.gfk.com/enus/insights/press-release/daily-contact-lenses-surpassmonthlies-in-us-sales-account-for-38-of-market/

5. Bergenske P, Long B, Dillehay S, et al. Long-term clinical results: 3 years of up to 30-night continuous wear of lotrafilcon A silicone hydrogel and daily wear of low-Dk/t hydrogel lenses. Eye Contact Lens 2007;33(2):74-80.

6. Dillehay SM. Does the level of available oxygen impact comfort in contact lens wear?: A review of the literature. Eye Contact Lens 2007;33(3):148-55.

7. Dillehay SM, Miller MB. Performance of lotrafilcon B silicone hydrogel contact lenses in experienced lowDk/t daily lens wearers. Eye Contact Lens 2007;33(6 Pt 1):272-7.

8. Papas EB. The significance of oxygen during contact lens wear. Cont Lens Anterior Eye 2014;37(6):394-404.

9. Grant T, Tang A. A Survey of contact lens wearers and eye care professionals on satisfaction with a new smartsurface silicone hydrogel daily disposable contact lens. Clin Optom 2020;12:9-15. 